\title{
Monoclonal antibody to the six-transmembrane epithelial antigen of prostate 4 promotes apoptosis and inhibits proliferation and glucose uptake in human adipocytes
}

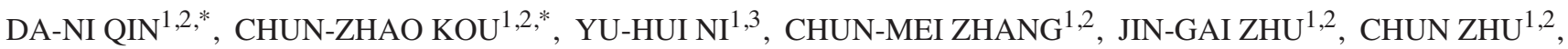 \\ YAN-PING WANG ${ }^{1,2}$, GUAN-ZHONG ZHU ${ }^{1,2}$, CHUNMEI-SHI $^{1,2}$, CHEN-BO JI $^{1,2}$ and XI-RONG GUO ${ }^{1,2}$ \\ ${ }^{1}$ Department of Pediatrics, Nanjing Maternal and Child Health Hospital of Nanjing Medical University, Nanjing 210004; \\ ${ }^{2}$ Institute of Pediatrics, Nanjing Medical University, Nanjing 210029, P.R. China; \\ ${ }^{3}$ Allele Biotechnology and Pharmaceuticals Inc., San Diego, CA 92121, USA
}

Received August 9, 2010; Accepted September 27, 2010

DOI: 10.3892/ijmm_00000528

\begin{abstract}
We previously identified the six-transmembrane epithelial antigen of prostate (STEAP) 4 as a novel plasma membrane protein that is up-regulated in obese patients and may play a significant role in the development of human obesity. In this study, a STEAP4-specific antibody was used to characterize the biological functions of the STEAP4 protein in human adipocytes. Cell viability assays (Trypan Blue exclusion), CCK-8 assays and cell cycle analysis showed that the STEAP4 antibody inhibited pre-adipocyte proliferation. Morphological observations by electron microscopy and confocal laser microscopy, annexin V-FITC labeling, caspase- 3 and caspase- 8 activity assays as well as data from quantitative real-time RT-PCR (qPCR) further determined that the STEAP4 antibody could promote apoptosis in pre-adipocytes. Based on quantitative Oil Red O staining and the expression profiles of specific markers, we demonstrated that the STEAP4 antibody did not affect adipogenesis, but the 2-deoxy-d-[ $\left.{ }^{3} \mathrm{H}\right]$-glucose uptake tests showed that it induced the insulin-stimulated glucose uptake in mature human adipocytes. In conclusion, our results demonstrated that the STEAP4 antibody does not influence human adipocyte differentiation, but it is likely that the STEAP4 protein regulates proliferation and apoptosis and plays an important role in modulating the insulin sensitivity of human adipocytes.
\end{abstract}

Correspondence to: Dr Xi-Rong Guo or Dr Chen-Bo Ji, Department of Pediatrics, Nanjing Maternal and Child Health Hospital of Nanjing Medical University, No.123 Tianfei Road, Nanjing 210004, P.R. China

E-mail:xrguo@njmu.edu.cn

E-mail: chenboji@njmu.edu.cn

*Contributed equally

Key words: six-transmembrane epithelial antigen of prostate 4 antibody, adipocyte, apoptosis, obesity, insulin sensitivity

\section{Introduction}

The prevalence of obesity has reached epidemic proportions in developed countries and is a growing problem in developing countries. The consequences of obesity include insulin resistance, type 2 diabetes, hypertension, hyperlipidemia, cardiac infarction and alterations in growth factors and other hormones that are important for the development of arteriosclerosis (1-4). Obesity is a multifactorial disease resulting from interactions between susceptibility genes, psychosocial and environmental factors. Although the environmental and psychosocial factors are important, there is considerable evidence for the significant role of genetic factors in the pathogenesis of obesity. Hence, identifying the genes involved in obesity would help to elucidate the underlying mechanisms of the disease.

The six-transmembrane epithelial antigen of prostate 4 (STEAP4, also called STAMP2, TIARP) $(5,6)$, a member of the six-transmembrane protein family, has attracted considerable interest in the research on obesity and energy metabolism. Moldes et al reported that STEAP4 expression is strongly induced during adipose conversion in 3T3-L1 adipocytes (7). In mice, STEAP4 expression in white adipose tissue (WAT) was induced by obesity (5). STEAP4 knockout mice were reported to display impairment of insulin-stimulated glucose transport in adipocytes (5). Furthermore, clinical research revealed that adipose STEAP4 expression has clearly increased in obese people from Sweden (8). Our group previously showed that the expression of STEAP4 in human adipocytes, could be regulated by the adipokines such as TNF- $\alpha$, interleukin-6 (IL-6) and leptin (9). Together, these results suggest a close relationship between STEAP4 and obesity or obesity-related insulin resistance. However, the exact functional properties of STEAP4 in the development of obesity have not been elucidated thus far.

In the current study, we aimed to investigate the functional role of STEAP4 in adipocytes. Immunocytochemical and Western blot analyses have previously shown that STEAP4 is localized at the plasma membranes of adipose cells (10). Monoclonal antibodies generated recently were demonstrated to bind to the extracellular domains of hSTEAP4, suggesting 
that these antibodies could be functional to promote or inhibit the functions of STEAP4 (11). Therefore, we used an antiSTEAP4 monoclonal antibody to target its extracellular domain and found that the antibody could significantly induce apoptosis in human adipocytes and inhibit cellular proliferation and glucose uptake.

\section{Materials and methods}

Antibody. The mouse monoclonal antibody against STEAP4 was purchased from Lifespan Biosciences (Seattle, WA, USA). Antibodies were dissolved in phosphate-buffered saline (PBS) containing 5\% trehalose and were purified through protein $\mathrm{G}$ beads.

Cell culture and treatment. Human pre-adipocytes (ScienCell Research Laboratories, San Diego, CA, USA) were maintained in Dulbecco's modified Eagle's medium (DMEM; Gibco-BRL, Carlsbad, CA, USA) containing 10\% fetal bovine serum (FBS; Gibco-BRL), $100 \mathrm{U} / \mathrm{ml}$ penicillin and $50 \mu \mathrm{g} / \mathrm{ml}$ streptomycin at $37^{\circ} \mathrm{C}$ in $5 \% \mathrm{CO}_{2}$. In order to induce differentiation, confluent human pre-adipocytes (day 0 ) were subsequently cultured in serum-free DMEM containing $50 \mathrm{nM}$ insulin, $100 \mathrm{nM}$ dexamethasone, $0.5 \mathrm{mM}$ 3-isobutyl-1-methylxanthine, and $100 \mu \mathrm{M}$ rosiglitazone. The medium was changed every 2 days for the first 4 days. Thereafter, the medium was replaced by serum-free DMEM containing $50 \mathrm{nM}$ insulin and $100 \mathrm{nM}$ dexamethasone, which was changed every 2 days until lipid droplets had accumulated (days 14-17). Fat accumulation was assessed by staining formalin-fixed cells with Oil Red O.

Seventeen days after the induction of differentiation, more than $90 \%$ of the cells had the morphological and biochemical properties of adipocytes, the cells used for the experiments. After treatment with STEAP4 antibody for different periods of time $(2,4,8,11,14$, or 17 days $)$, the adipocytes were collected and prepared for further study.

Viability assessments by trypan blue exclusion. Cells were collected by centrifugation at $1500 \mathrm{rcf}$ for $8 \mathrm{~min}$. After resuspension in PBS, the dying or dead cells were identified by positive staining with $0.2 \%$ trypan blue (Sigma-Aldrich). Means and SDs of antibody-induced dead cells were calculated based on three independent experiments.

CCK-8 assay. Adipocytes (8x102/well) were seeded in 96well plates and maintained in 10\% FBS DMEM for $24 \mathrm{~h}$ until they were adherent. Media were then changed to DMEM supplemented with $0.5 \%$ FBS. When the cells grew to $80 \%$ confluency, they were starved in serum-free DMEM for $12 \mathrm{~h}$ before treatment with or without the STEAP4 antibody at various concentrations for $24 \mathrm{~h}$ in DMEM supplemented with $0.5 \%$ FBS. Cell growth was also monitored over the course of seven consecutive days with or without treatment with the STEAP4 antibody. The proliferation rate was assessed by using the Cell Counting Kit-8 (CCK-8; Dojindo, Kumamoto, Japan) according to the manufacturer's protocol. In brief, the CCK-8 solution $(5 \mu \mathrm{l})$ was added to each well and incubated for $2 \mathrm{~h}$ before absorbance values at 450 and $650 \mathrm{~nm}$ were recorded by an ELISA reader, and the difference between these values was recorded as the optical density (OD).
Cell cycle assay. Cells $\left(2 \times 10^{6} / 750 \mathrm{~mm}^{2}\right)$ were cultured in DMEM with $10 \%$ FBS. Once adhered, the cells were washed with PBS and starved in serum-free DMEM for $24 \mathrm{~h}$ to synchronize the cells. Cell cycle analysis was initiated at various time points $(0,6,12,18,24,30,36,42$, and $48 \mathrm{~h})$ after replacement of the starvation medium with complete medium. Cultured cells were harvested using trypsin/EDTA and washed twice with PBS. Aliquots of $2 \times 10^{6}$ cells were centrifuged, fixed in $70 \%$ ethanol overnight in $-20^{\circ} \mathrm{C}$, and then stained with $500 \mathrm{ml}$ propidium iodide (PI) solution (100 mg/ml RNase and $50 \mathrm{mg} / \mathrm{ml}$ PI in 1X PBS). Labeled cells were analyzed using BD FACScan and CellQuest software (BD Biosciences, San Jose, CA, USA).

Oil Red $O$ staining and quantification. Human adipocytes were cultured in 12-well plates and induced to become mature adipocytes. For Oil Red O staining, the culture medium was removed and the cells were washed twice with PBS. The cells were then fixed with $4 \%$ formalin/PBS for $10 \mathrm{~min}$ at room temperature. After fixation, the cells were washed with PBS again and stained with $0.6 \%(\mathrm{w} / \mathrm{v})$ filtered Oil Red O solution (60\% isopropanol, $40 \%$ water) for $45 \mathrm{~min}$ at room temperature. After washing three times, the cells were observed using an optical microscope and were photographed.

To quantitate the amount of Oil Red O staining, the cells were washed and by elution with isopropanol, and the OD of the solution was measured at $520 \mathrm{~nm}$ (12).

Quantitative real-time RT-PCR ( $P P C R)$. Total RNA was extracted using TRIzol reagent (Invitrogen, Carlsbad, CA, USA) from adipocytes and quantified spectrophotometrically at $260 \mathrm{~nm}$. cDNA was synthesized from $1 \mu \mathrm{g}$ of total RNA using an AMV Reverse Transcriptase Kit (Promega A3500; Madison, WI, USA). qPCR (SYBR Green method) was performed in an Applied Biosystems 7500 Sequence Detection System (ABI 7500 SDS; Foster City, CA, USA) following the manufacturer's protocol. Briefly, samples were incubated at $95^{\circ} \mathrm{C}$ for $10 \mathrm{~min}$ for initial denaturation, and then subjected to $40 \mathrm{PCR}$ cycles, each consisting of $95^{\circ} \mathrm{C}$ for $15 \mathrm{sec}$ and $60^{\circ} \mathrm{C}$ for $1 \mathrm{~min}$. $18 \mathrm{~S}$ or $\beta$-actin was used as a reference to obtain the relative fold-change for target samples using the comparative CT method calculated by the formula: 2 [CT (reference gene) - CT (target gene)]. The sequences of the primers used are shown in Table I.

Electron microscopy. Cells were cultured in serum-free DMEM for $24 \mathrm{~h}$ and exposed to STEAP4 antibody solutions or left without treatment for $24 \mathrm{~h}$. Adipocytes were then digested by trypsin, washed in fresh PBS ( $\mathrm{pH} 7.4)$ and fixed in $2.5 \%$ glutaraldehyde $/ 4 \%$ paraformaldehyde in the same buffer. The cells were then washed in $0.1 \mathrm{M}$ cacodylate buffer, post-fixed with $1 \%$ osmium tetroxide/ $1.5 \%$ potassium ferrocyanide for $1 \mathrm{~h}$, washed in water, stained with $1 \%$ aqueous uranyl acetate for $30 \mathrm{~min}$, and then dehydrated through a graded series of ethanol to $100 \%$. The samples were then infiltrated and embedded in TAAB Epon (Marivac Canada Inc., St. Laurent, Canada). Ultrathin sections (60 nm) were cut on a Reichert Ultracut-S microtome, placed onto copper grids stained with uranyl acetate and lead citrate, 
Table I. Sequences of primer sets used in qPCR.

\begin{tabular}{lll}
\hline Gene & \multicolumn{1}{c}{ Forward primer $\left(5^{\prime}-3^{\prime}\right)$} & \multicolumn{1}{c}{ Reverse primer $\left(5^{\prime}-3^{\prime}\right)$} \\
\hline PPAR $\gamma$ & CGAAGACATTCCATTCACAAGA & GCTTTATCTCCACAGACACGA \\
LPL & CGCTCCATTCATCTCTTCATC & TTGCTGCTTCTTTTGGCTCT \\
CEBPa & AGGTTTCCTGCCTCCTTCC & AGCCCCAAGTCCCTATGTTT \\
$18 \mathrm{~s}$ & CGGGTCGGGAGTGGGTAAT & AGTCGCCGTGCCTACCAT \\
Bax & TGTCTGTCTTGTCCCCTTCC & ACCTTGAGCACCAGTTTGCT \\
Bcl-2 & TCCATGTCTTTGGACAACCA & CTCCACCAGTGTTCCCATCT \\
B-actin & TCACCCACACTGTGCCCATCTACGA & CAGCGGAACCGCTCATTGCCAATGG
\end{tabular}

and examined on a JEOL JEM-1010 transmission electron microscope at an accelerating voltage of $80 \mathrm{kV}$.

Confocal laser microscopy and flow cytometry. Cells were cultured in FBS-free DMEM for $24 \mathrm{~h}$ and then with or without exposure to STEAP4 antibodies for $48 \mathrm{~h}$ to induce apoptosis. Cells were harvested using trypsin/EDTA, washed with PBS, resuspended in $1 \mathrm{ml}$ binding buffer, and stained with $10 \mu \mathrm{l}$ annexin V-FITC and $10 \mu \mathrm{l}$ PI at room temperature for $10 \mathrm{~min}$ (Biovision, CA, USA). The FITC and PI fluorescent signals were analyzed by examining smears under a confocal laser microscope and by a flow cytometer.

Measurement of caspase-3 and caspase-8 activity. After inducing apoptosis, cells were collected and washed with PBS. Activities of caspase- 3 and caspase- 8 were assayed using a commercially available kit (Sigma, St. Louis, MO, USA) according to the manufacturer's protocol. Additional assays were performed using inhibitors included in the kit.

Glucose uptake. 2-Deoxy-d-[3 $\mathrm{H}]$-glucose (CIC, Beijing, China) uptake was assayed as described previously (13). Briefly, cells were cultured and were induced to become mature adipocytes in 6-well plates. After becoming fully mature, the cells were cultured in serum-free medium, followed by a $24 \mathrm{~h}$ incubation with or without STEAP4 antibody $(1: 250)$ at $37^{\circ} \mathrm{C}$ and then by a 30 min incubation with or without insulin $(100 \mathrm{nmol} / \mathrm{l})$ at $37^{\circ} \mathrm{C}$. Labeled 2-deoxy$\mathrm{d}-\left[{ }^{3} \mathrm{H}\right]$-glucose was added to a final concentration of $2 \mu \mathrm{Ci} / \mathrm{ml}$. After $10 \mathrm{~min}$ at $37^{\circ} \mathrm{C}$, the reaction was terminated by washing three times with ice-cold PBS supplemented with $10 \mathrm{mmol} / \mathrm{l}$ d-glucose. The cells were solubilized by the addition of $200 \mu 1$ of $1 \mathrm{~mol} / \mathrm{l} \mathrm{NaOH}$ to each well, and aliquots of the cell lysate were transferred to scintillation vials for radioactivity measurement. The remainder was used for protein assays.

Statistical analysis. All data are presented as the means \pm SE. Data were analyzed by one-way ANOVA or the Student's t-test using the SPSS 15.0 statistical package. A P-value of $<0.05$ was considered to be statistically significant.

\section{Results}

Effect of the STEAP4 antibody on pre-adipocyte cell viability. The trypan blue exclusion assay, the most common test for

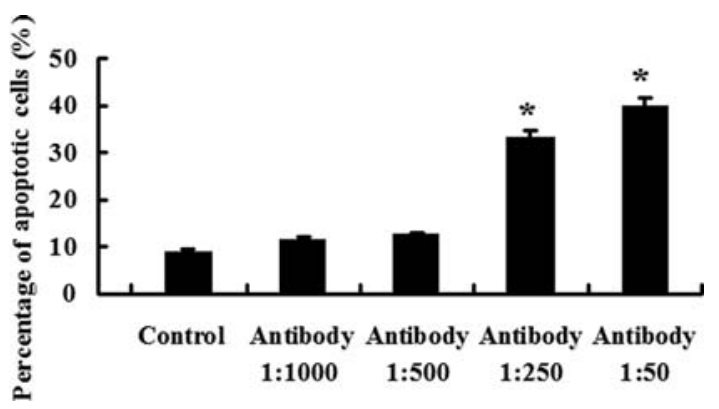

Figure 1. Effects of the STEAP4 antibodies on viability. The trypan blue exclusion assay was used for the quantification of dead cells. Four antibody dilutions were tested (1:50, 1:250, 1:500 and 1:1000 dilutions), and a serumdeprived group with antibody dilution liquid was used as a control. When the cells grew to $80 \%$ confluency, they were serum starved for $12 \mathrm{~h}$ before exposure to the STEAP4 antibody or left without treatment for $48 \mathrm{~h}$. Results are representative of three separate experiments, ${ }^{*} \mathrm{P}<0.05$.

cell viability, was used for the quantification of dead cells. We initially performed experiments with different antibody concentrations but we found that antibody dilutions do not affect the cell activity (data not shown). We used the antibody concentration of $0.5 \mathrm{mg} / \mathrm{ml}$ and assessed the effect of four antibody dilutions [1:50 (0.01 mg/ml), 1:250 $(0.002 \mathrm{mg} / \mathrm{ml})$, $1: 500(0.001 \mathrm{mg} / \mathrm{ml})$, and $1: 1000(0.0005 \mathrm{mg} / \mathrm{ml})]$ on the viability of the pre-adipocytes using samples from the serumdeprived only and blank groups as controls $\left({ }^{*} \mathrm{P}<0.05\right)$. The antibody dilutions of 1:50 and 1:250 promoted cell death, while the antibody dilutions of 1:500 and 1:1000 did not have any significant effect on cell viability (Fig. 1).

Effect of the STEAP4 antibody on cell proliferation. The CCK-8 assay was used to evaluate the proliferation of preadipocytes (Fig. 2A and B) with or without STEAP4 antibody treatment. A decreased growth rate was observed in cells exposed to different concentrations of the STEAP4 antibody compared with control cells $\left({ }^{*} \mathrm{P}<0.05,{ }^{* *} \mathrm{P}<0.001\right)$. The STEAP4 antibody also affected the cell cycle. Flow cytometric analysis of cell cycle distribution (Fig. 2C) detected a significantly lower percentage of the cells exposed to the STEAP4 antibody in the S phase of the cell cycle $\left({ }^{*} \mathrm{P}<0.05\right.$ compared to control cells).

Effect of the STEAP4 antibody on cell differentiation. Next, we investigated whether the STEAP4 antibodies could affect 

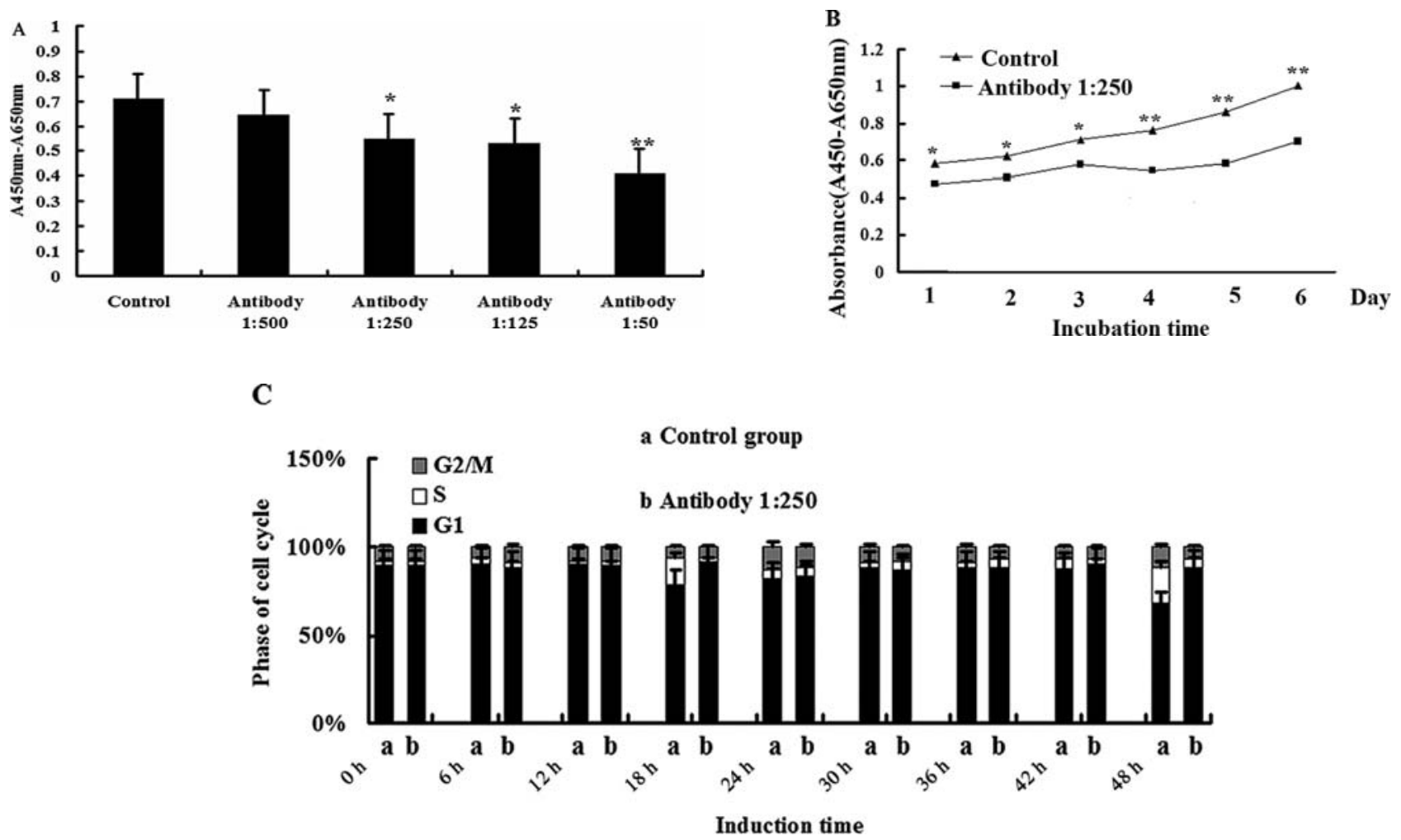

Figure 2. Effects of STEAP4 antibodies on cell proliferation. The CCK-8 kit was used to assess cell proliferation (A and B). (A) Cell proliferation was monitored after a $24 \mathrm{~h}$ treatment with different STEAP4 antibody dilutions. (B) Cell growth was monitored in pre-adipocytes treated with the STEAP4 antibody (1:250 diliution) over the course of seven consecutive days. (C) The cell cycle stages of pre-adipocytes treated with the STEAP4 antibody (1:250 dilution) or control were monitored by flow cytometry. Results from both assays showed that the STEAP4 antibody inhibited the proliferation of human preadipocytes. Data are presented as means $\pm \mathrm{SE}$ of 6 experiments, ${ }^{*} \mathrm{P}<0.05,{ }^{* *} \mathrm{P}<0.001$.

human pre-adipocyte differentiation. As a control, preadipocytes cells were treated with PBS. As shown in Fig. 3A, the number and size of lipid droplets were not significantly different between STEAP4 treated groups and the control groups during the 17 days following induction of differentiation (x400). The cells exposed to STEAP4 antibody (1:250) had higher lipid levels than the control group based on Oil Red $\mathrm{O}$ staining, but the difference did not reach statistical significance (Fig. 3B, P>0.05). Furthermore, we analyzed the expression of adipocyte-specific molecular markers, including the CCAAT enhancer-binding protein a (CEBPa), peroxisome proliferator-activated receptor $\gamma$ (PPAR $\gamma$ ) and LPL, the expressions of which are known to be up-regulated during human pre-adipocyte differentiation. The expression levels of these marker genes detected by qPCR in human pre-adipocyte cells treated with the STEAP4 antibody (1:250) on days $0,4,8,12$, and 17 were similar to the control cells at the same time points (Fig. 3C, P>0.05).

Effect of the STEAP4 antibody on cell apoptosis. Electron microscopy, which is the best method for morphologically observing differentiating nuclei and organelles was used to confirm apoptosis. As shown in Fig. 4A (panels a, b and c), there were no typical morphological changes in the control cells, but obvious apoptotic morphological changes were observed in the cells exposed to STEAP4 antibody solutions for $48 \mathrm{~h}$ (Fig. 4A, panels $\mathrm{d}$ and e). Panel a shows the typical ultrastructure of an untreated human pre-adipocyte (x8000) characterized by a well-preserved plasma membrane. Abundant microvilli are seen on the surface and the nucleus contains a nucleolus and euchromatin. Panel b shows an untreated human pre-adipocyte cultured in FBS-free DMEM for $48 \mathrm{~h}$ to induce apoptosis. The cytoplasm shows numerous normal organelles including mitochondria and a dictyosome. Panels $d$ and e show the early stage of apoptosis in cells treated with STEAP4 antibody at 1:500, and 1:250 dilutions, respectively (x8000). Most organelles in the cytoplasm had lost some of their individual characteristics, and few microvilli were seen on the cell surface. At a higher magnification in Fig. 4B (x30000), two typical effects of mitochondrial damage, swelling and pyknosis, caused by the STEAP4 antibody can be found. Recovery from mitochondrial swelling is possible while recovery from mitochondrial pyknosis is not, implying that, with the increase in the concentration of STEAP4 antibodies, the cells tended to undergo apoptosis (Fig. 4C). These results demonstrated that apoptosis in human pre-adipocyte cells was induced by STEAP4 antibodies at dilutions of 1:250 and 1:50 and could perhaps be induced with higher concentrations of the antibody.

Cell binding studies with annexin V-FITC were used to observe apoptosis in the sedimented cell smears under a fluorescence laser scanning confocal microscope (x200). Preadipocyte cells were cultured in FBS-free DMEM with or without STEAP4 antibody solutions for $48 \mathrm{~h}$, and binding studies with annexin V-FITC showed that the antibodies promoted serum deprivation-induced apoptosis in pre- 
A
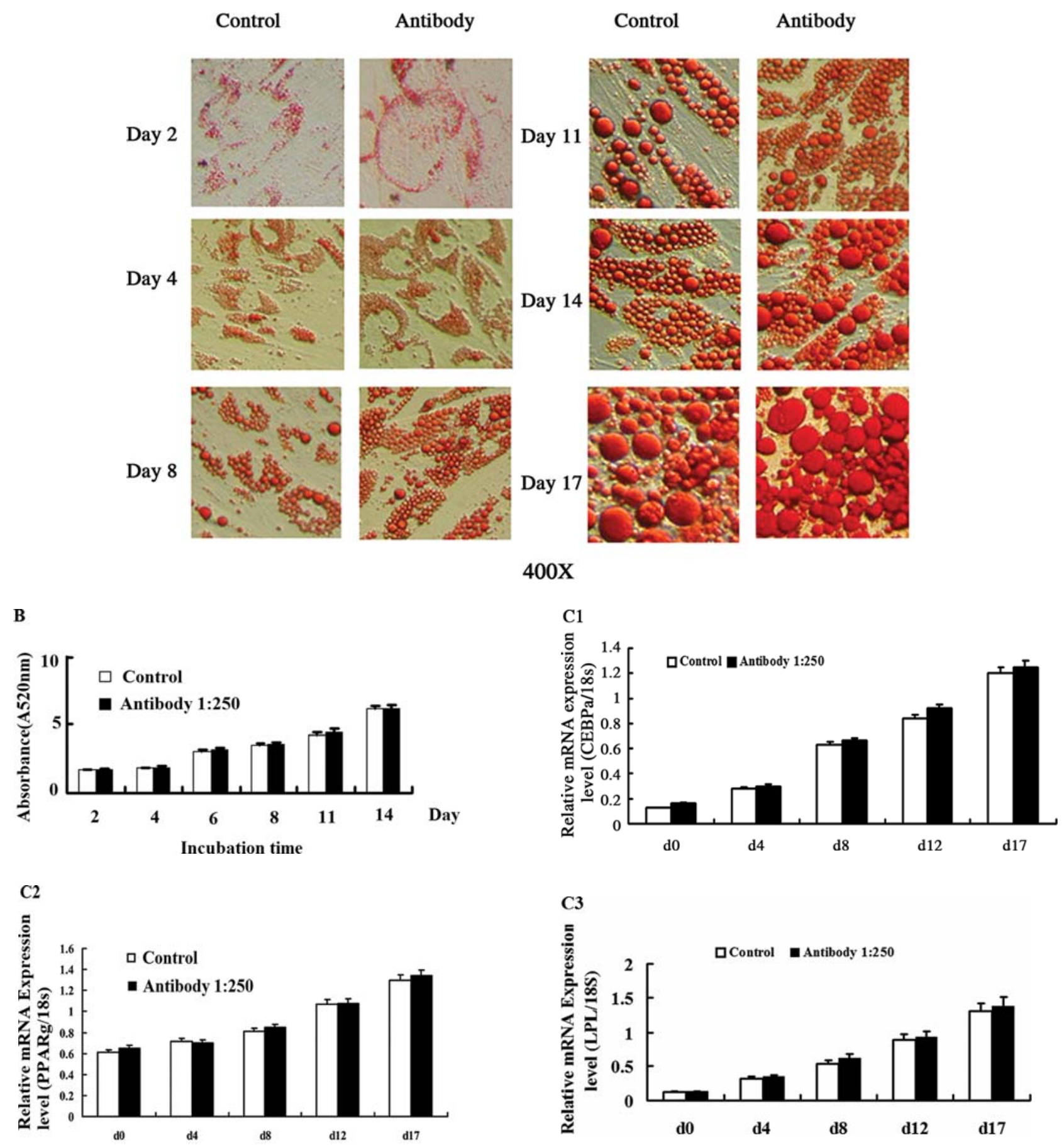

Figure 3. Effects of the STEAP4 antibody on cell differentiation. (A) Human pre-adipocytes cells were exposed to the STEAP4 antibody at the dilution of 1:250 or control with antibody dilution and stimulated to differentiate over 17 days. On days 2, 4, 8,11,14 and 17, the cells were fixed and stained with Oil Red $\mathrm{O}$ to visualize lipids. (B) The cells were then destained from the Oil Red O by isopropanol elution after the cells had been washed, and the optical densities of the solutions were measured at $520 \mathrm{~nm}$. (C) Expressions of adipocyte-specific molecular markers were analyzed by qPCR at various time points during the stimulation of differentiation $(0,4,8,12$ and 17 days). No differences in expression levels of these markers were found between cells with or without STEAP4 antibody treatment. Data are presented as means \pm SE of 6 experiments, $P>0.05$.

adipocytes (Fig. 4D, P<0.05). Red fluorescence represents cells in apoptosis, and yellow signals represent those in early apoptosis. Thus, the results showed that the increase in antibody concentrations gradually increased red fluorescent apoptotic cells.

The Bcl-2 family proteins have been considered to act as upstream factors in the apoptotic machinery; the Bcl-2 families of genes can either positively or negatively regulate programmed cell death. Although Bcl-2 is a proto-oncogene and can suppress apoptosis, it can also inhibit apoptosis, and the $\mathrm{Bcl}-2 / \mathrm{Bax}$ ratio has been correlated with cell survival (14). Thus, we used the $\mathrm{Bax} / \mathrm{Bcl}-2$ gene expression ratio to quantitatively assess cellular apoptosis. In order to determine whether $\mathrm{Bax}$ and $\mathrm{Bcl}-2$ are involved in cell apoptosis induced 
A

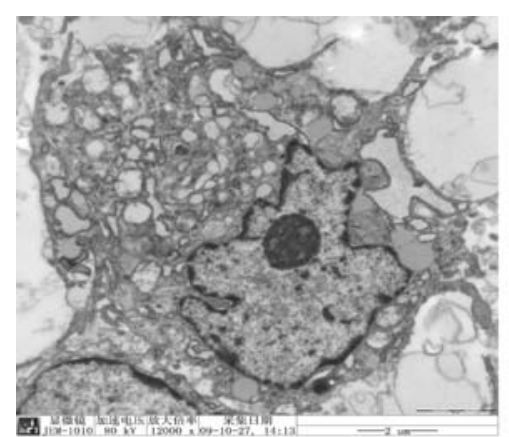

a. Control group

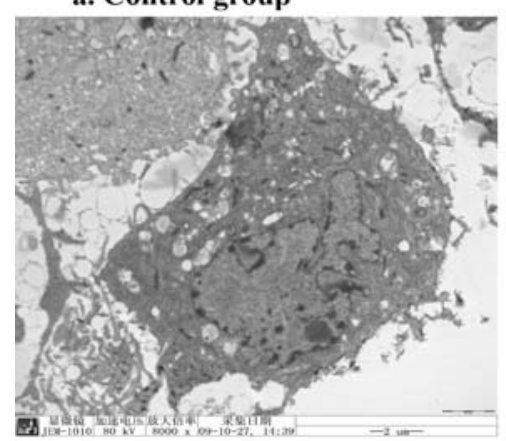

c. Antibody 1:500 group

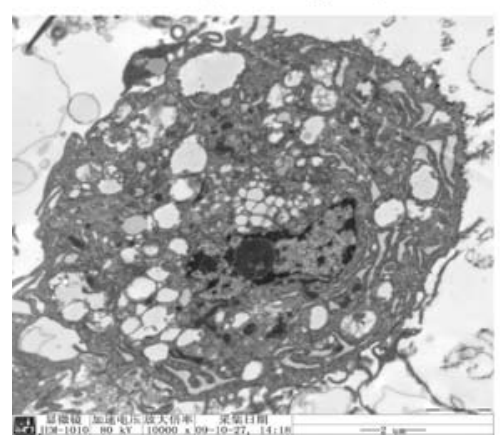

B

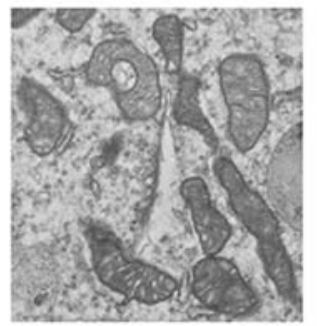

Control group

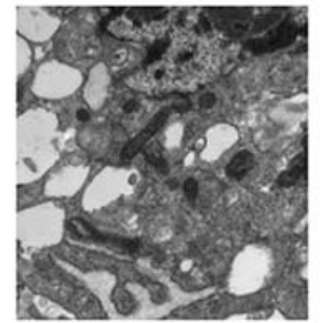

Antibody 1:250 group

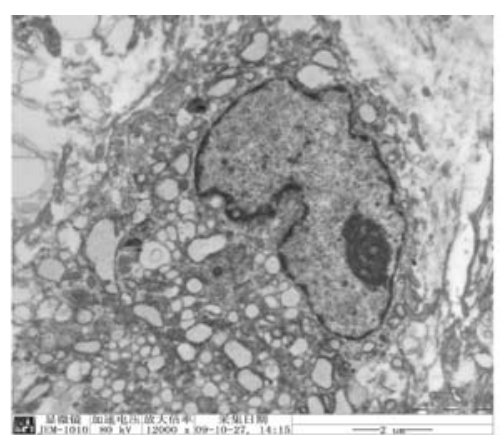

b. Antibody 1:1000 group

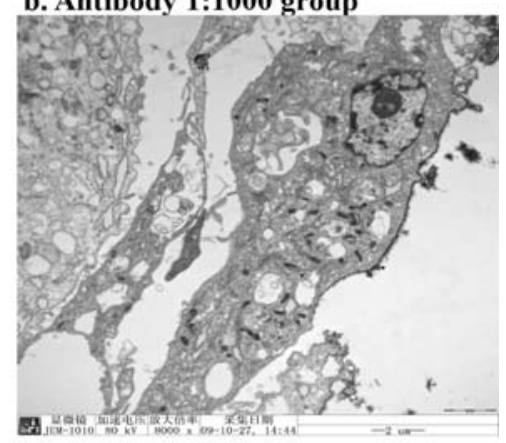

d. Antibody 1:250 group

C

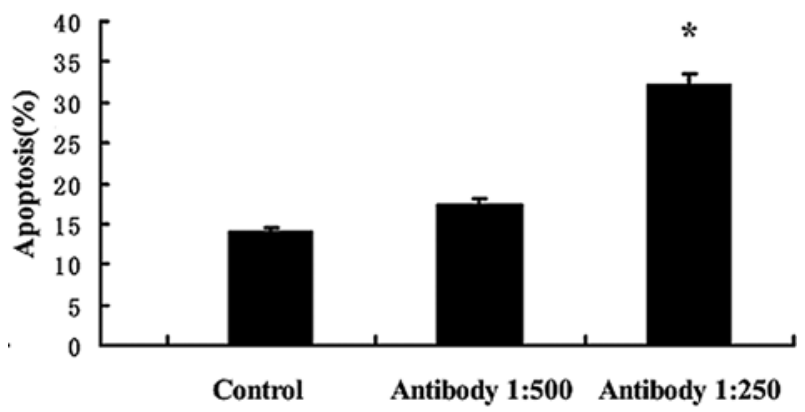

$30000 \mathrm{X}$

Figure 4. A-C, Effects of STEAP4 antibodies on cell apoptosis.

by STEAP4 antibodies, we used qPCR to evaluate their mRNA levels normalized to those of $B$-actin in STEAP4 antibody-treated adipocytes and control cells. Fig. 4E shows that with STEAP4 antibody dilutions of 1:50, 1:250 and 1:500, the $\mathrm{Bax} / \mathrm{Bcl}-2$ ratios were significantly greater than those in serum-deprived adipocytes and control cells ( $\left.{ }^{* *} \mathrm{P}<0.001\right)$.

The key effector molecules in the apoptotic pathway are the caspases, a family of cysteine proteases, which are activated through proteolytic cleavage in both the mitochondrial- and death receptor-mediated apoptotic pathways (15). Caspase- 3 and caspase- 8 play critical roles in cell apoptosis, and were confirmed to be induced by STEAP4 antibodies that promoted serum deprivation-induced apoptosis (Fig. 4F, $\mathrm{P}<0.001)$.

Effects of the STEAP4 antibody on basal and insulinstimulated glucose uptake in human adipocytes. A 2-deoxyd-glucose uptake assay was been used to examine whether 
D

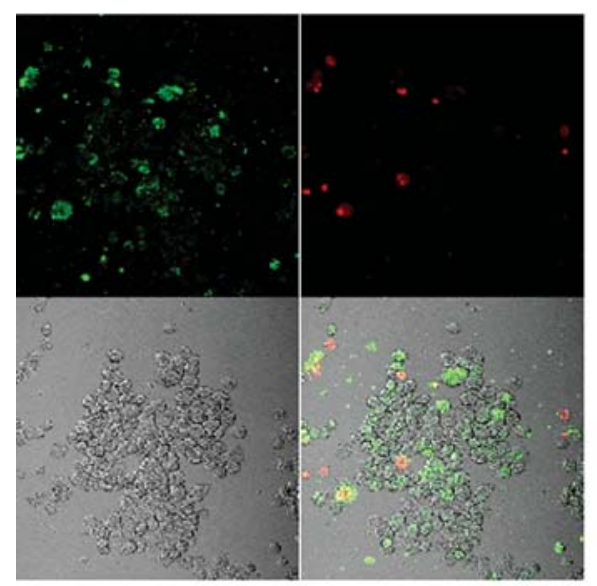

a. Control group

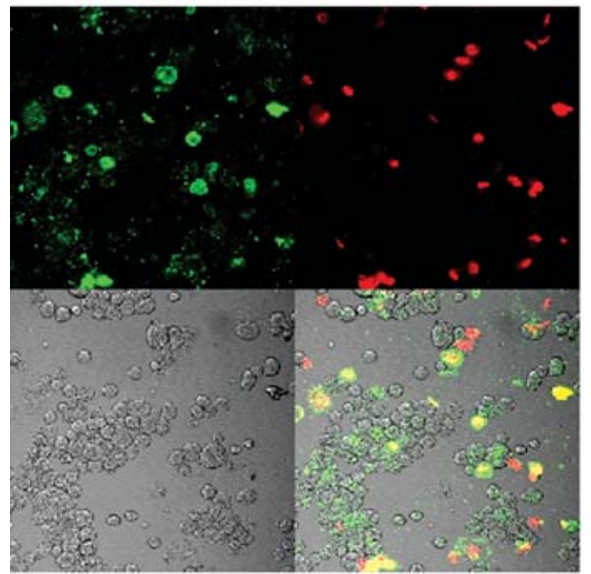

c. Antibody 1:250 group

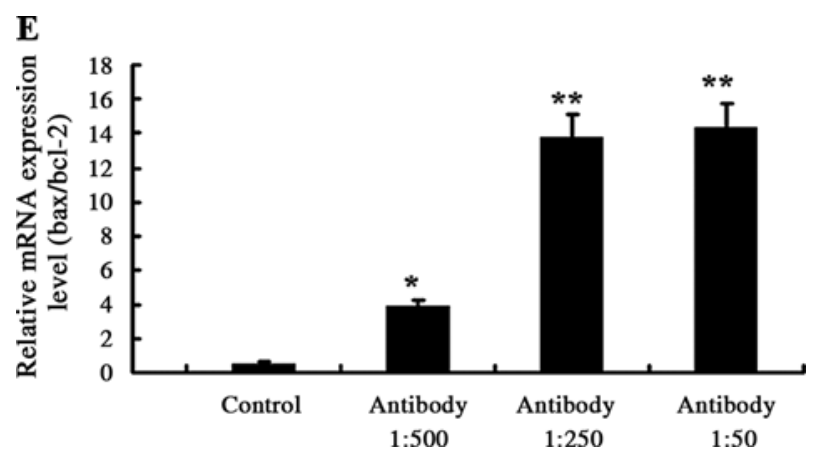

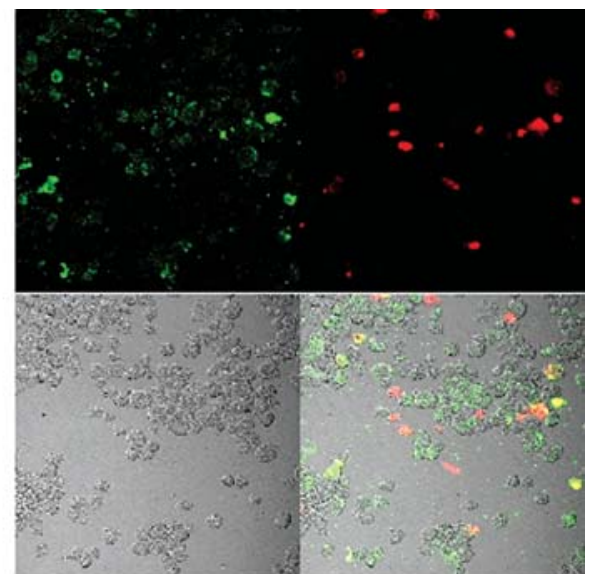

b. Antibody 1:500 group

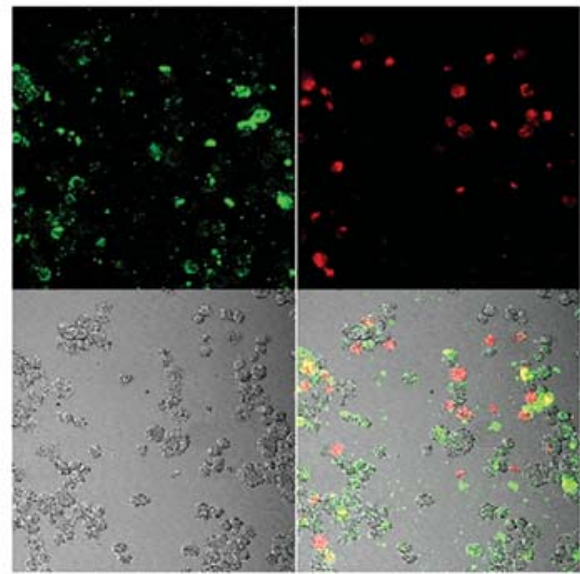

d. Antibody 1: 50 group

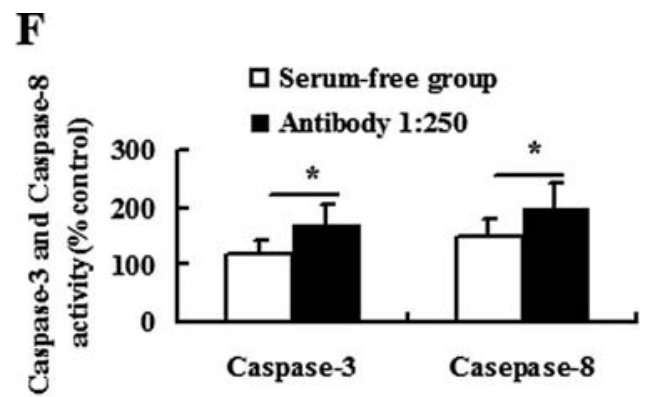

Figure 4. Effects of STEAP4 antibodies on cell apoptosis. The effect of the STEAP4 antibody on cell morphology was detected by (A) electron microscopy (x8000) and (D) confocal microscopy (x200). (B) Mitochondrial changes were observed at a higher magnification (x30000) by electron microscopy. (C) Apoptosis was assayed by binding of annexin V-FITC. Representative images from two independent experiments are shown. (E) Effects of the STEAP4 antibody on the mRNA expression levels of Bax and Bcl-2 in human pre-adipocytes. The mRNA levels of Bax, Bcl-2 were analyzed using qPCR and normalized to $\beta$-actin levels. The ratios of Bax/Bcl-2 represent the levels of apoptosis. Data are presented as means $\pm \mathrm{SE}$ of 6 experiments, ${ }^{* *} \mathrm{P}<0.01$. $(\mathrm{F}) \mathrm{Cells}$ were cultured in FBS-free DMEM for $48 \mathrm{~h}$ to induce apoptosis (with or without antibody 1:250), and caspase-3 and caspase- 8 activities were assessed.

STEAP4 antibodies affected the glucose uptake of mature adipocytes. As shown in Fig. 5, upon exposure to STEAP4 antibodies, basal glucose uptake in the adipocytes was similar to that observed in the controls. However, after insulin stimulation, a significant difference was found between the two groups. In the STEAP4 antibody treated adipocyte cells, insulin-stimulated glucose uptake was much lower than that in the controls.

\section{Discussion}

Obesity is a multifactorial disease resulting from interactions between susceptibility genes, psychosocial and environmental factors. Understanding the genes involved in the development of obesity is important for the development of new therapies that directly target molecular mechanisms underlying the condition. STEAP4, a novel obesity-related 


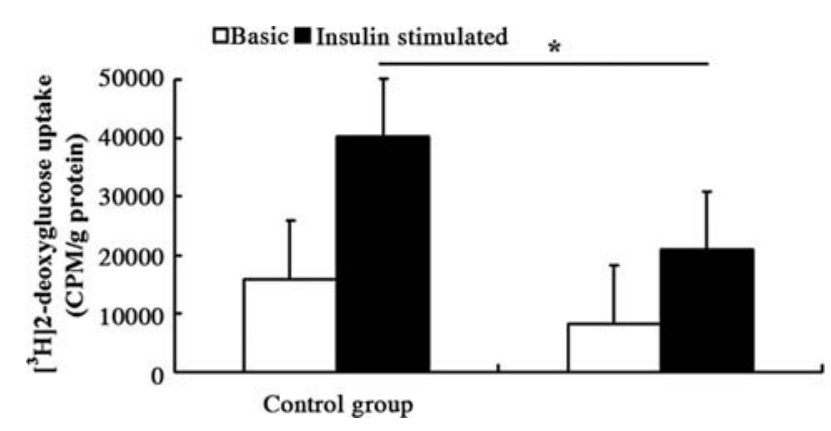

Figure 5. Effects of the STEAP4 antibody on basal and insulin-stimulated glucose uptake in human adipocytes. Human pre-adipocytes with or without the STEAP4 antibody (1:250) were induced to become mature adipocytes. After serum starvation, the mature adipocytes were incubated with (black columns) or without (white columns) $100 \mathrm{nmol} / 1$ insulin for $30 \mathrm{~min}$ followed by measurement of the 2-deoxy-D- $\left[{ }^{3} \mathrm{H}\right]$-glucose uptake. Values represent the means \pm SD from three independent experiments. ${ }^{*} \mathrm{P}<0.05$ vs. insulin-stimulated control.

gene, has been screened in our laboratory by suppression subtractive hybridization (16) of the omental adipose tissue in obese patients and normal-weight controls. In the present study, we used an anti-STEAP4 antibody to study the relationship between obesity and STEAP4. We found that the anti-STEAP4 monoclonal antibodies inhibited proliferation of human pre-adipocytes, promoted their apoptosis, and affected their insulin sensitivity, but not their differentiation. We thus, hypothesize that these antibodies may provide a new treatment option for obesity.

Adipose tissue, as a metabolic and endocrine organ, plays an essential role in the regulation of energy balance (17). Accordingly, adipocytes are emerging as a potential therapeutic target for obesity, type 2 diabetes, cardiovascular disease and cancer. The mass of adipose tissue reflects the number and average volume of adipocytes, in particular the balance between cell acquisition and cell loss $(18,19)$. Therefore, the proliferation of adipocyte precursors and their differentiation into mature adipocytes combined with the apoptosis of pre-adipocytes, all contribute to the development of obesity in mammals $(20,25)$. Studies on mouse models $(5,6)$ have shown that STEAP4 plays an important role in the coordinated regulation of nutrient and inflammatory responses and that it is associated with insulin sensitivity in mice. We studied the effect of the STEAP4 protein on cell growth by two methods. The CCK- 8 assay demonstrated that preadipocytes treated with the STEAP4 antibody grew much more slowly than control cells. Cell cycle analysis by flow cytometry showed a reduced percentage of S-phase cells in STEAP4 antibody-treated pre-adipocytes. Together these data demonstrated that the STEAP4 antibody reduces the proliferation of human pre-adipocyte cells. We also found that the STEAP4 antibody does not affect the differentiation of human pre-adipocyte cells. As obesity is the result of an expansion of individual adipocytes and an increase in the number of adipocytes, we believe that STEAP4 may stimulate the growth rate of human pre-adipocytes as a signaling molecule to promote obesity.

In addition, a predictive structural analysis of the STEAP4 protein suggests a potential function of STEAP4 in energy metabolism and electron transport $(5,7)$. Other members of the STEAP family have been characterized as metalloreductases capable of facilitating the cellular uptake of iron and copper, which are required in both glucose and lipid metabolism (4). As these functions are important for supporting survival of the cells, it is likely that exposure to the STEAP4 antibody would promote apoptosis in these cells by altering these functions. Another explanation of the ability of the STEAP4 antibody to promote cell death is by its effects on the mitochondria. By electron microscopy analysis, we observed two types of cellular injuries, mitochondrial swelling and pyknosis. Mitochondria are at the center of the regulatory processes for apoptosis in that they can release apoptosis-related proteins and cause cell death through both caspase-dependent and caspase-independent mechanisms (20). The release of these apoptogenic proteins have been shown to be modulated by members of the Bcl-2 family, which are major regulators of the mitochondria-initiated caspase activation pathway, consistent with their role in apoptosis (15).

Pre-adipocytes, whose functions are to uptake glucose and convert it into lipids then store it in the cells, play an important role in obesity. Furthermore, the results from the 2-deoxy-d- $\left[{ }^{3} \mathrm{H}\right]$ glucose uptake assay indicated that the STEAP4 antibody caused a reduction in the insulin sensitivity of mature human adipocytes by reducing the insulin-stimulated glucose uptake, suggesting that STEAP4 may play a role in the insulin sensitivity of adipocytes. Collectively, our results indicate that the STEAP4 antibody could cause mitochondrial dysfunction in adipocytes, which may be responsible for the development of STEAP4 antibody-induced insulin resistance. Although our results suggest that the STEAP4 protein affects the insulin signaling pathway, the mechanism is not clear yet and should be further studied.

In conclusion, our data provide evidence that the STEAP4 antibodies could induce significant effects on adipocytes by inhibiting cell proliferation, promoting cellular apoptosis, but without affecting differentiation of the cells. The antibodies may also affect the insulin-signaling pathway in adipocytes. Collectively, these findings may bring new insights into the treatment of obesity and related complications.

\section{Acknowledgements}

This project was supported by grants from the National Natural Science Foundation of China (No. 30772364), the Natural Science Foundation of Jiangsu Province, China (No. BK2007230), the Foundation of the Ministry of Education, China (No. 20070312001), and the Graduate Students Research and Innovation Plan of Jiangsu Province (No. CX09B_256Z).

\section{References}

1. Spiegelman BM and Flier JS: Obesity and the regulation of energy balance. Cell 104: 531-543, 2001.

2. Goodman E, Slap GB and Huang B: The public health impact of socioeconomic status on adolescent depression and obesity. Am J Public Health 93: 1844-1850, 2003.

3. Porkka KP, Helenius MA and Visakorpi T: Cloning and characterization of a novel six-transmembrane protein STEAP2, expressed in normal and malignant prostate. Lab Invest 82: 1573-1582, 2002.

4. Ohgami RS, Campagna DR, McDonald A and Fleming MD: The Steap proteins are metalloreductases. Blood 108: 1388-1394, 2006. 
5. Korkmaz CG, Korkmaz KS, Kurys P, Elbi C, Wang L, Klokk TI, Hammarstrom C, Troen G, Svindland A, Hager Gl and Saatcioglu F: Molecular cloning and characterization of STAMP2, an androgen-regulated six transmembrane protein that is overexpressed in prostate cancer. Oncogene 24: 4934-4945, 2005.

6. Wellen KE, Fucho R, Gregor MF, Furuhashi M, Morgan C, Lindstad T, Vaillancourt E, Gorgun CZ, Saatcioglu F and Hotamisligil GS: Coordinated regulation of nutrient and inflammatory responses by STAMP2 is essential for metabolic homeostasis. Cell 129: 537-548, 2007.

7. Moldes M, Lasnier F, Gauthereau X, Klein C, Pairault J, Feve B and Chambaut-Guerin AM: Tumor necrosis factor-alphainduced adipose-related protein (TIARP), a cell-surface protein that is highly induced by tumor necrosis factor-alpha and adipose conversion. J Biol Chem 276: 33938-33946, 2001.

8. Arner P, Stenson BM, Dungner E, Naslund E, Hoffstedt J, Ryden $M$ and Dahlman I: Expression of six transmembrane protein of prostate 2 in human adipose tissue associates with adiposity and insulin resistance. J Clin Endocrinol Metab 93: 2249-2254, 2008.

9. Chen X, Zhu C, Ji C, Zhao Y, Zhang C, Chen F, Gao C, Zhu J, Qian L and Guo X: STEAP4, a gene associated with insulin sensitivity, is regulated by several adipokines in human adipocytes. Int J Mol Med 25: 361-367, 2010.

10. Zhang CM, Chi X, Wang B, Zhang M, Ni YH, Chen RH, Li XN and Guo XR: Downregulation of STEAP4, a highly-expressed TNF-alpha-inducible gene in adipose tissue, is associated with obesity in humans. Acta Pharmacol Sin 29: 587-592, 2008.

11. Tamura T and Chiba J: Production of antibodies against multipass membrane proteins expressed in human tumor cells using dendritic cell immunization. J Biomed Biotechnol 2009: 673098, 2009.

12. Murase Y, Kobayashi J, Nohara A, Asano A, Yamaaki N, Suzuki K, Sato H and Mabuchi H: Raloxifene promotes adipocyte differentiation of 3T3-L1 cells. Eur J Pharmacol 538: $1-4,2006$.

13. Ceddia RB, Somwar R, Maida A, Fang X, Bikopoulos G and Sweeney G: Globular adiponectin increases GLUT4 translocation and glucose uptake but reduces glycogen synthesis in rat skeletal muscle cells. Diabetologia 48: 132-139, 2005.

14. Gross A, Yin XM, Wang K, Wei MC, Jockel J, Milliman C, Erdjument-Bromage H, Tempst P and Korsmeyer SJ: Caspase cleaved BID targets mitochondria and is required for cytochrome $\mathrm{c}$ release, while BCL-XL prevents this release but not tumor necrosis factor-R1/Fas death. J Biol Chem 274: 1156-1163, 1999.
15. Brooks C and Dong Z: Regulation of mitochondrial morphological dynamics during apoptosis by Bcl-2 family proteins: a key in Bak. Cell Cycle 6: 3043-3047, 2007.

16. Diatchenko L, Lau YF, Campbell AP, Chenchik A, Moqadam F, Huang B, Lukyanov S, Lukyanov K, Gurskaya N, Sverdlov ED and Siebert PD: Suppression subtractive hybridization: a method for generating differentially regulated or tissue-specific cDNA probes and libraries. Proc Natl Acad Sci USA 93: 6025-6030, 1996.

17. Rosen ED and Spiegelman BM: Adipocytes as regulators of energy balance and glucose homeostasis. Nature 444: 847-853, 2006.

18. Furuyashiki T, Nagayasu H, Aoki Y, Bessho H, Hashimoto T, Kanazawa $\mathrm{K}$ and Ashida $\mathrm{H}$ : Tea catechin suppresses adipocyte differentiation accompanied by down-regulation of PPARgamma2 and C/EBPalpha in 3T3-L1 cells. Biosci Biotechnol Biochem 68: 2353-2359, 2004.

19. Brook CG, Lloyd JK and Wolf OH: Relation between age of onset of obesity and size and number of adipose cells. Br Med J 2: 25-27, 1972 .

20. Prins JB and O'Rahilly S: Regulation of adipose cell number in man. Clin Sci (Lond) 92: 3-11, 1997.

21. Prins JB, Walker NI, Winterford CM and Cameron DP: Apoptosis of human adipocytes in vitro. Biochem Biophys Res Commun 201: 500-507, 1994.

22. Sorisky A, Magun R and Gagnon AM: Adipose cell apoptosis: death in the energy depot. Int J Obes Relat Metab Disord 24: S3-S7, 2000.

23. Della-Fera MA, Qian H and Baile CA: Adipocyte apoptosis in the regulation of body fat mass by leptin. Diabetes Obes Metab 3: 299-310, 2001.

24. Margareto J, Aguado M, Oses-Prieto JA, Rivero I, Monge A, Aldana I, Marti A and Martinez JA: A new NPY-antagonist strongly stimulates apoptosis and lipolysis on white adipocytes in an obesity model. Life Sci 68: 99-107, 2000.

25. Della-Fera MA, Li C and Baile CA : Resistance to IP leptininduced adipose apoptosis caused by high-fat diet in mice. Biochem Biophys Res Commun 303: 1053-1057, 2003. 